\title{
Social Regulations Predispose People to Complete Vaccination for Vaccine-Preventable Diseases
}

\author{
Jiro Takeuchi, ${ }^{1}$ Masashi Goto, ${ }^{1}$ Takashi Kawamura ${ }^{1}$ and Atsushi Hiraide ${ }^{2}$ \\ ${ }^{1}$ Kyoto University Health Service, Kyoto, Kyoto, Japan \\ ${ }^{2}$ Department of Acute Medicine, Kinki University Faculty of Medicine, Osaka-Sayama, Osaka, Japan
}

\begin{abstract}
Japan experienced measles outbreaks in both 2006 and 2007 mainly among university students. Improvement of vaccine coverage against vaccine-preventable viral infections is the prime task for preventing outbreaks of viral infections. To elucidate the promoting factors for complete vaccination against measles, rubella, mumps, and varicella-zoster viruses, we conducted a case-control study among single university students in Japan. Information on vaccinations and clinico-demographical factors were collected using a self-administered questionnaire and a photocopy of the Maternal and Child Health Handbook. Logistic regression analysis was performed to estimate odds ratios (ORs) and their $95 \%$ confidence intervals $(\mathrm{Cls})$ for two-time vaccination against measles and rubella viruses as mandatory vaccinations and at least one-time vaccination against mumps and varicella-zoster viruses as optional vaccinations. A total of 1,370 (744 medical, 508 paramedical, and 118 pharmaceutical) students were invited to participate, 960 $(70.1 \%)$ of whom were enrolled in the study. Students aged $<20$ years had a greater propensity for measles and rubella vaccinations (OR $7.8[95 \% \mathrm{Cl}, 5.1-11.8]$ and OR 6.1 [95\% Cl, 3.7-10.0], respectively) compared with those aged $\geq 20$ years. Students with a history of living over-seas for 1 month or longer were more likely to complete vaccination for measles (OR 4.4 [95\% Cl, 1.4-13.5] compared with those without such history. This significantly high vaccination coverage was attributed to the measles-rubella catch-up campaign by the Japanese government and the immunization regulations by foreign countries. These findings suggest that social regulations would predispose people to complete vaccination.
\end{abstract}

Keywords: coverage; Maternal and Child Health Handbook; university students; vaccine; vaccine-preventable diseases

Tohoku J. Exp. Med., 2014 November, 234 (3), 183-187. C 2014 Tohoku University Medical Press

\section{Introduction}

Japan experienced measles outbreaks in both 2006 and 2007 mainly among university students. Improvement of vaccine coverage against vaccine-preventable viral infections is the prime task for preventing outbreaks of viral infections among college students in Japan. To obtain sufficient antibody titers against measles or rubella among college students, a recent study indicated that two or more doses of vaccination are needed (Takeuchi et al. 2013).

Vaccination behavior is known to be determined by social and domestic factors such as legal obligations, municipal provision of services, access to the health care system, economic and educational status, family structure, and concerns about vaccine safety (Marks et al. 1979; Wood et al. 1995; Holt et al. 1996; Suarez et al. 1997; Morrow et al. 1998; Yusuf et al. 2002; Bardenheier et al. 2004; Allred et al. 2005; Gust et al. 2008; Smith et al. 2011). However, vaccinee factors associated with complete vaccination against measles, rubella, mumps, and varicella-zoster viruses among university students have not been evaluated in detail.

In Japan, the Maternal and Child Health $(\mathrm{MCH})$ Handbook is issued by local governments and filled out by medical professionals mainly at pre- and post-natal periodic health checkups and on-demand perinatal medical office visits, according to the MCH Law. The MCH Handbook can provide reliable information on immunization status, past history, and perinatal history (Takayanagi et al. 1993; Reich et al. 2008; Centers for Disease Control and Prevention 2009; Osaki et al. 2009; Nakamura 2010). Among participating university students, this study therefore analyzed information obtained from both the $\mathrm{MCH}$ Handbook and a self-administered questionnaire.

\section{Methods}

We carried out a case-control study at a single institution in Japan. The 2008 all-year and 2009 first-year students of the faculties

Received May 27, 2014; revised and accepted September 26, 2014. Published online October 16, 2014; doi: 10.1620/tjem.234.183. Correspondence: Takashi Kawamura, Kyoto University Health Service, Yoshida-Honmachi, Sakyo-ku, Kyoto, Kyoto 606-8501, Japan. e-mail: kawax@kuhp.kyoto-u.ac.jp 
of Medicine and Pharmaceutical Sciences at Kyoto University were invited to participate. Information on vaccination and candidate factors were collected from a self-administered questionnaire and a photocopy of each participants' MCH Handbook. The MCH Handbook included dates of measles, rubella, mumps, and varicella-zoster vaccinations, a history of infection with those viruses, birthplace, gestational week, birth weight, history of neonatal asphyxia, history of infectious diseases requiring hospitalization, steroid or immunosuppressive drug usage, and the presence of siblings. We applied logistic regression analysis to estimate odds ratios (ORs) and $95 \%$ confidence intervals (CIs) for two-time vaccination against measles and rubella viruses and at least one-time vaccination against mumps and varicella-zoster viruses. We did not include variables in multivariate models if observations were available from fewer than half of the participants or if the number of individual selections for a dichotomized option in a question was less than 10. We performed analyses using Stata 10.0 software (Stata Corporation, College Station, TX, USA). All tests of significance were 2-tailed, and values of $P<0.05$ were considered significant.

We obtained individual written, informed consent from all study participants prior to enrollment. This investigation was approved by the Ethics Committee of Kyoto University Graduate School of Medicine.

\section{Results}

A total of 1,370 students (medical, $n=744$; paramedical, $n=508$; pharmaceutical, $n=118$ ) were invited to participate, of whom 960 of them (70.1\%) with information on vaccination history were enrolled in the study (Table 1).

We did not include history of neonatal asphyxia or steroid or immunosuppressive drug usage in the multivariate models because of the small number of observations or selections. Associations between two-time vaccination against measles and rubella viruses and clinico-demographic factors are summarized in Table 1. In the multivariate analyses, female students were significantly more likely than males to have received measles vaccination (OR 1.5 [95\% CI, 1.0-2.2]). Students $<20$ years old showed a notably higher OR for measles or rubella vaccination than older students (OR 7.8 [95\% CI, 5.1-11.8] and OR 6.1 [95\% CI, 3.7-10.0], respectively). Students with a history of living overseas for 1 month or longer were significantly more likely to have undergone measles vaccination (OR 4.4 [95\% CI, 1.4-13.5]).

Associations between voluntary vaccination against mumps and varicella-zoster viruses and clinico-demographic factors are summarized in Table 2. Preterm birth or low birth weight were associated with a significantly increased probability of varicella vaccination (OR 2.5 [95\% CI, 1.2-5.2]). Absence of siblings was associated with a significantly increased probability of varicella vaccination (OR 1.9 [95\% CI, 1.1-3.2]).

\section{Discussion}

We elucidated factors associated with complete vaccinations for measles, rubella, mumps, and varicella among university students by analyzing perinatal histories, subse- quent life history, and family factors. Significant vaccination-promoting factors were female sex and history of living overseas for 1 month or longer for measles, age $<20$ years for both measles and rubella, and preterm birth or low birth weight and absence of siblings for varicella.

Of note, age $<20$ years was associated with a marked enhancement of measles and rubella vaccinations. This could be attributed to the governmental measles-rubella catch-up campaign that promoted measles-rubella combined vaccination at 17-18 years old (third-year high school students) promoted throughout Japan in 2008-2013 (Centers for Disease Control and Prevention 2009; Takeuchi et al. 2014). Likewise, vaccination has been required by the government of Japan for students with an intention to stay abroad for 1 month or longer. Conversely, vaccination for mumps was enforced until 1993 as a combined measlesrubella-mumps vaccine, so younger ages would yield a somewhat decreased OR. Based on these findings, social regulation appears to have a strong effect on vaccination coverage.

The observed high proportion of complete vaccination for varicella among students with preterm birth or low birth weight may be attributed to parental anxiety regarding babies encountering health concerns early in life, which might prompt them to ensure their children undergo vaccination (Rosenstock et al. 1988).

Potential limitations of the current analysis must be acknowledged when interpreting these findings. First, selection bias might have been present if students with a poor vaccination history hesitated to participate in the study, and would result in an overestimation of the proportion of vaccination behaviors. Second, we could not establish a solid consensus based on our findings, because of the limited number of participants and the imbalanced distribution of outcomes and predictors. For example, only $17 \%$ of students received two or more vaccinations for rubella. Third, recall bias of parents responding to the self-administered questionnaire may have distorted the results of the current analysis. A study of measles, rubella, mumps, and varicella among Japanese medical students has previously shown that use of a questionnaire alone is insufficient to provide reliable information (Manago et al. 2004). We therefore used the MCH Handbook to obtain objective information about infection and immunization. Despite these various several limitations, the present results will allow discussion of the clinico-demographic factors of vaccinees.

In conclusion, this analysis suggests that social regulation may predispose individuals to complete vaccination. 
Table 1. Association between routine vaccination against measles and rubella viruses and clinico-demographic factors.

\begin{tabular}{|c|c|c|c|c|c|c|c|c|}
\hline & \multicolumn{4}{|c|}{ Measles } & \multicolumn{4}{|c|}{ Rubella } \\
\hline & $\begin{array}{l}\geq 2 \text { doses } \\
(n=255)\end{array}$ & $\begin{array}{l}<2 \text { doses } \\
(n=705)\end{array}$ & $\begin{array}{c}\text { Crude odds } \\
\text { ratio } \\
\left(95 \% \mathrm{CI}^{\ddagger}\right)\end{array}$ & $\begin{array}{c}\text { Adjusted } \\
\text { odds ratio* } \\
\left(95 \% \mathrm{CI}^{\ddagger}\right) \\
(n=655)\end{array}$ & $\begin{array}{l}\geq 2 \text { doses } \\
(n=162)\end{array}$ & $\begin{array}{l}<2 \text { doses } \\
(n=792)\end{array}$ & $\begin{array}{c}\text { Crude odds } \\
\text { ratio } \\
\left(95 \% \mathrm{CI}^{\ddagger}\right)\end{array}$ & $\begin{array}{c}\text { Adjusted } \\
\text { odds ratio* } \\
\left(95 \% \mathrm{CI}^{\ddagger}\right) \\
(n=655)\end{array}$ \\
\hline \multicolumn{9}{|l|}{ Sex } \\
\hline Male & $\begin{array}{l}133 / 255 \\
(52.2 \%)\end{array}$ & $\begin{array}{l}409 / 705 \\
(58.0 \%)\end{array}$ & 1.0 & 1.0 & $\begin{array}{r}83 / 162 \\
(51.2 \%)\end{array}$ & $\begin{array}{l}458 / 792 \\
(57.8 \%)\end{array}$ & 1.0 & 1.0 \\
\hline Female & $\begin{array}{l}122 / 255 \\
(47.8 \%)\end{array}$ & $\begin{array}{l}296 / 705 \\
(42.0 \%)\end{array}$ & $\begin{array}{c}1.3 \\
(0.95-1.7)\end{array}$ & $\begin{array}{c}1.5 \\
(1.0-2.2)\end{array}$ & $\begin{array}{r}79 / 162 \\
(48.8 \%)\end{array}$ & $\begin{array}{l}334 / 792 \\
(42.2 \%)\end{array}$ & $\begin{array}{c}1.1 \\
(0.78-1.6)\end{array}$ & $\begin{array}{c}1.4 \\
(0.94-2.2)\end{array}$ \\
\hline \multicolumn{9}{|l|}{ Age } \\
\hline$\geq 20$ years & $\begin{array}{r}43 / 255 \\
(16.9 \%)\end{array}$ & $\begin{array}{l}410 / 705 \\
(58.2 \%)\end{array}$ & 1.0 & 1.0 & $\begin{array}{r}26 / 162 \\
(16.1 \%)\end{array}$ & $\begin{array}{l}423 / 792 \\
(53.4 \%)\end{array}$ & 1.0 & 1.0 \\
\hline$<20$ years & $\begin{array}{l}212 / 255 \\
(83.1 \%)\end{array}$ & $\begin{array}{l}295 / 705 \\
(41.8 \%)\end{array}$ & $\begin{array}{c}6.5 \\
(4.5-9.4)\end{array}$ & $\begin{array}{c}7.8 \\
(5.1-11.8)\end{array}$ & $\begin{array}{l}136 / 162 \\
(83.9 \%)\end{array}$ & $\begin{array}{l}369 / 792 \\
(46.6 \%)\end{array}$ & $\begin{array}{c}4.0 \\
(2.5-6.4)\end{array}$ & $\begin{array}{c}6.1 \\
(3.7-10.0)\end{array}$ \\
\hline \multicolumn{9}{|l|}{ Birth place } \\
\hline Non-metropolitan areas & $\begin{array}{l}166 / 232 \\
(71.5 \%)\end{array}$ & $\begin{array}{l}300 / 432 \\
(69.4 \%)\end{array}$ & 1.0 & 1.0 & $\begin{array}{l}108 / 145 \\
(74.5 \%)\end{array}$ & $\begin{array}{l}358 / 519 \\
(69.0 \%)\end{array}$ & 1.0 & 1.0 \\
\hline Metropolitan areas ${ }^{\dagger}$ & $\begin{array}{r}66 / 232 \\
(28.5 \%)\end{array}$ & $\begin{array}{l}132 / 432 \\
(30.6 \%)\end{array}$ & $\begin{array}{c}0.90 \\
(0.64-1.3)\end{array}$ & $\begin{array}{c}0.96 \\
(0.64-1.4)\end{array}$ & $\begin{array}{r}37 / 145 \\
(25.5 \%)\end{array}$ & $\begin{array}{l}161 / 519 \\
(31.0 \%)\end{array}$ & $\begin{array}{c}0.75 \\
(0.48-1.2)\end{array}$ & $\begin{array}{c}0.8 \\
(0.52-1.3)\end{array}$ \\
\hline \multicolumn{9}{|l|}{ Gestational week/birth weight } \\
\hline Full-term and normal birth weight & $\begin{array}{l}218 / 244 \\
(89.3 \%)\end{array}$ & $\begin{array}{l}430 / 477 \\
(90.1 \%)\end{array}$ & 1.0 & 1.0 & $\begin{array}{l}138 / 154 \\
(89.6 \%)\end{array}$ & $\begin{array}{l}509 / 566 \\
(89.9 \%)\end{array}$ & 1.0 & 1.0 \\
\hline Preterm/low birth weight & $\begin{array}{l}15 / 244 \\
(6.2 \%)\end{array}$ & $\begin{array}{c}19 / 477 \\
(4.0 \%)\end{array}$ & $\begin{array}{c}1.6 \\
(0.79-3.1)\end{array}$ & $\begin{array}{c}1.7 \\
(0.73-3.8)\end{array}$ & $\begin{array}{c}7 / 154 \\
(4.6 \%)\end{array}$ & $\begin{array}{l}27 / 566 \\
(4.8 \%)\end{array}$ & $\begin{array}{c}1.1 \\
(0.44-2.8)\end{array}$ & $\begin{array}{c}1.1 \\
(0.43-2.8)\end{array}$ \\
\hline Postterm birth/macrosomia & $\begin{array}{c}11 / 244 \\
(4.5 \%)\end{array}$ & $\begin{array}{c}28 / 477 \\
(5.9 \%)\end{array}$ & $\begin{array}{c}0.77 \\
(0.38-1.6)\end{array}$ & $\begin{array}{c}0.83 \\
(0.37-1.9)\end{array}$ & $\begin{array}{c}9 / 154 \\
(5.8 \%)\end{array}$ & $\begin{array}{c}30 / 566 \\
(5.3 \%)\end{array}$ & $\begin{array}{c}1.2 \\
(0.54-2.9)\end{array}$ & $\begin{array}{c}1.3 \\
(0.56-3.0)\end{array}$ \\
\hline \multicolumn{9}{|l|}{ Neonatal asphyxia } \\
\hline No & $\begin{array}{c}90 / 95 \\
(94.7 \%)\end{array}$ & $\begin{array}{l}135 / 146 \\
(92.5 \%)\end{array}$ & 1.0 & & $\begin{array}{c}62 / 66 \\
(93.9 \%)\end{array}$ & $\begin{array}{l}163 / 175 \\
(93.1 \%)\end{array}$ & 1.0 & \\
\hline Yes & $\begin{array}{c}5 / 95 \\
(5.3 \%)\end{array}$ & $\begin{array}{c}11 / 146 \\
(7.5 \%)\end{array}$ & $\begin{array}{c}0.68 \\
(0.23-2.0)\end{array}$ & - & $\begin{array}{c}4 / 66 \\
(6.1 \%)\end{array}$ & $\begin{array}{c}12 / 175 \\
(6.9 \%)\end{array}$ & $\begin{array}{c}1.0 \\
(0.28-3.5)\end{array}$ & - \\
\hline \multicolumn{9}{|c|}{ Infectious diseases requiring hospitalization } \\
\hline No & $\begin{array}{l}201 / 244 \\
(82.4 \%)\end{array}$ & $\begin{array}{l}429 / 481 \\
(89.2 \%)\end{array}$ & 1.0 & 1.0 & $\begin{array}{l}132 / 153 \\
(86.3 \%)\end{array}$ & $\begin{array}{l}497 / 571 \\
(87.0 \%)\end{array}$ & 1.0 & 1.0 \\
\hline Yes & $\begin{array}{r}43 / 244 \\
(17.6 \%)\end{array}$ & $\begin{array}{r}52 / 481 \\
(10.8 \%)\end{array}$ & $\begin{array}{c}1.3 \\
(0.97-1.8)\end{array}$ & $\begin{array}{c}1.6 \\
(0.96-2.7)\end{array}$ & $\begin{array}{r}21 / 153 \\
(13.7 \%)\end{array}$ & $\begin{array}{r}74 / 571 \\
(13.0 \%)\end{array}$ & $\begin{array}{c}1.0 \\
(0.68-1.5)\end{array}$ & $\begin{array}{c}1.0 \\
(0.58-1.8)\end{array}$ \\
\hline \multicolumn{9}{|c|}{ Steroid or immunosuppressive drug usage } \\
\hline No & $\begin{array}{l}243 / 246 \\
(98.8 \%)\end{array}$ & $\begin{array}{l}519 / 523 \\
(99.2 \%)\end{array}$ & 1.0 & & $\begin{array}{l}153 / 155 \\
(98.7 \%)\end{array}$ & $\begin{array}{l}606 / 611 \\
(99.2 \%)\end{array}$ & 1.0 & \\
\hline Yes & $\begin{array}{c}3 / 246 \\
(1.2 \%)\end{array}$ & $\begin{array}{c}4 / 523 \\
(0.8 \%)\end{array}$ & $\begin{array}{c}1.6 \\
(0.36-7.2)\end{array}$ & - & $\begin{array}{c}2 / 155 \\
(1.3 \%)\end{array}$ & $\begin{array}{c}5 / 611 \\
(0.8 \%)\end{array}$ & $\begin{array}{c}1.7 \\
(0.28-10.2)\end{array}$ & - \\
\hline \multicolumn{9}{|l|}{$\geq 1$ month stay abroad } \\
\hline No & $\begin{array}{l}229 / 244 \\
(93.9 \%)\end{array}$ & $\begin{array}{l}458 / 477 \\
(96.0 \%)\end{array}$ & 1.0 & 1.0 & $\begin{array}{l}143 / 153 \\
(93.5 \%)\end{array}$ & $\begin{array}{l}546 / 563 \\
(97.0 \%)\end{array}$ & 1.0 & 1.0 \\
\hline Yes & $\begin{array}{l}15 / 244 \\
(6.1 \%)\end{array}$ & $\begin{array}{c}19 / 477 \\
(4.0 \%)\end{array}$ & $\begin{array}{c}2.5 \\
(1.2-5.5)\end{array}$ & $\begin{array}{c}4.4 \\
(1.4-13.5)\end{array}$ & $\begin{array}{l}10 / 153 \\
(6.5 \%)\end{array}$ & $\begin{array}{l}17 / 563 \\
(3.0 \%)\end{array}$ & $\begin{array}{c}2.2 \\
(0.90-5.2)\end{array}$ & $\begin{array}{c}2.0 \\
(0.65-5.9)\end{array}$ \\
\hline \multicolumn{9}{|l|}{ Siblings } \\
\hline Yes & $\begin{array}{l}221 / 245 \\
(90.2 \%)\end{array}$ & $\begin{array}{l}421 / 483 \\
(87.2 \%)\end{array}$ & 1.0 & 1.0 & $\begin{array}{l}141 / 154 \\
(91.6 \%)\end{array}$ & $\begin{array}{l}500 / 573 \\
(87.3 \%)\end{array}$ & 1.0 & 1.0 \\
\hline No & $\begin{array}{l}24 / 245 \\
(9.8 \%)\end{array}$ & $\begin{array}{r}62 / 483 \\
(12.8 \%)\end{array}$ & $\begin{array}{c}0.74 \\
(0.45-1.2)\end{array}$ & $\begin{array}{c}0.63 \\
(0.34-1.2)\end{array}$ & $\begin{array}{l}13 / 154 \\
(8.4 \%)\end{array}$ & $\begin{array}{r}73 / 573 \\
(12.7 \%)\end{array}$ & $\begin{array}{c}0.63 \\
(0.34-1.2)\end{array}$ & $\begin{array}{c}0.56 \\
(0.27-1.2)\end{array}$ \\
\hline
\end{tabular}

Values are expressed as number or odds ratio.

Sample sizes vary between variables because of missing data.

*Adjusted for sex, age, birth place, gestational week/birth weight, infectious diseases requiring hospitalization, $\geq 1$ month stay abroad, and presence of siblings.

${ }^{\dagger}$ Cities with $\geq 500,000$ inhabitants.

${ }_{\star} \mathrm{CI}$, confidence interval. 
Table 2. Association between voluntary vaccination for mumps and varicella-zoster viruses and clinico-demographical factors.

\begin{tabular}{|c|c|c|c|c|c|c|c|c|}
\hline & \multicolumn{4}{|c|}{ Mumps } & \multicolumn{4}{|c|}{ Varicella-zoster } \\
\hline & $\begin{array}{l}\geq 1 \text { dose } \\
(n=552)\end{array}$ & $\begin{array}{l}<1 \text { dose } \\
(n=397)\end{array}$ & $\begin{array}{c}\text { Crude odds } \\
\text { ratio } \\
\left(95 \% \mathrm{CI}^{\ddagger}\right)\end{array}$ & $\begin{array}{c}\text { Adjusted } \\
\text { odds ratio* } \\
\left(95 \% \mathrm{CI}^{\ddagger}\right) \\
(n=660)\end{array}$ & $\begin{array}{l}\geq 1 \text { dose } \\
(n=247)\end{array}$ & $\begin{array}{l}<1 \text { dose } \\
(n=705)\end{array}$ & $\begin{array}{l}\text { Crude odds } \\
\text { ratio } \\
\left(95 \% \mathrm{CI}^{\ddagger}\right)\end{array}$ & $\begin{array}{c}\text { Adjusted } \\
\text { odds ratio* } \\
\left(95 \% \mathrm{CI}^{*}\right) \\
(n=660)\end{array}$ \\
\hline \multicolumn{9}{|l|}{ Sex } \\
\hline Male & $\begin{array}{l}331 / 552 \\
(60.0 \%)\end{array}$ & $\begin{array}{l}207 / 397 \\
(52.1 \%)\end{array}$ & 1.0 & 1.0 & $\begin{array}{l}127 / 247 \\
(51.4 \%)\end{array}$ & $\begin{array}{l}410 / 705 \\
(58.2 \%)\end{array}$ & 1.0 & 1.0 \\
\hline Female & $\begin{array}{l}221 / 552 \\
(40.0 \%)\end{array}$ & $\begin{array}{l}190 / 397 \\
(47.9 \%)\end{array}$ & $\begin{array}{c}0.72 \\
(0.56-0.94)\end{array}$ & $\begin{array}{c}0.87 \\
(0.62-1.2)\end{array}$ & $\begin{array}{l}120 / 247 \\
(48.6 \%)\end{array}$ & $\begin{array}{l}295 / 705 \\
(41.8 \%)\end{array}$ & $\begin{array}{c}0.72 \\
(0.25-2.1)\end{array}$ & $\begin{array}{c}1.2 \\
(0.81-1.8)\end{array}$ \\
\hline \multicolumn{9}{|l|}{ Age } \\
\hline$\geq 20$ years & $\begin{array}{l}283 / 552 \\
(51.3 \%)\end{array}$ & $\begin{array}{l}182 / 397 \\
(40.8 \%)\end{array}$ & 1.0 & 1.0 & $\begin{array}{l}102 / 247 \\
(41.3 \%)\end{array}$ & $\begin{array}{l}344 / 705 \\
(48.8 \%)\end{array}$ & 1.0 & 1.0 \\
\hline$<20$ years & $\begin{array}{l}269 / 552 \\
(48.7 \%)\end{array}$ & $\begin{array}{l}215 / 397 \\
(59.2 \%)\end{array}$ & $\begin{array}{c}0.68 \\
(0.52-0.89)\end{array}$ & $\begin{array}{c}0.73 \\
(0.52-1.0)\end{array}$ & $\begin{array}{l}145 / 247 \\
(58.7 \%)\end{array}$ & $\begin{array}{l}361 / 705 \\
(51.2 \%)\end{array}$ & $\begin{array}{c}0.66 \\
(0.21-2.0)\end{array}$ & $\begin{array}{c}1.4 \\
(0.94-2.0)\end{array}$ \\
\hline \multicolumn{9}{|l|}{ Birth place } \\
\hline Non-metropolitan areas & $\begin{array}{l}268 / 397 \\
(67.5 \%)\end{array}$ & $\begin{array}{l}198 / 267 \\
(74.2 \%)\end{array}$ & 1.0 & 1.0 & $\begin{array}{l}111 / 172 \\
(64.5 \%)\end{array}$ & $\begin{array}{l}354 / 490 \\
(72.2 \%)\end{array}$ & 1.0 & 1.0 \\
\hline Metropolitan $\operatorname{areas}^{\dagger}$ & $\begin{array}{l}129 / 397 \\
(32.5 \%)\end{array}$ & $\begin{array}{r}69 / 267 \\
(25.8 \%)\end{array}$ & $\begin{array}{c}1.4 \\
(0.98-2.0)\end{array}$ & $\begin{array}{c}1.4 \\
(0.97-2.0)\end{array}$ & $\begin{array}{r}61 / 172 \\
(35.5 \%)\end{array}$ & $\begin{array}{l}136 / 490 \\
(27.8 \%)\end{array}$ & $\begin{array}{c}1.2 \\
(0.33-4.4)\end{array}$ & $\begin{array}{c}1.4 \\
(0.96-2.1)\end{array}$ \\
\hline \multicolumn{9}{|l|}{ Gestational week/birth weight } \\
\hline Full-term and normal birth weight & $\begin{array}{l}388 / 422 \\
(90.3 \%)\end{array}$ & $\begin{array}{l}267 / 299 \\
(89.2 \%)\end{array}$ & 1.0 & 1.0 & $\begin{array}{l}156 / 183 \\
(85.2 \%)\end{array}$ & $\begin{array}{l}490 / 536 \\
(91.4 \%)\end{array}$ & 1.0 & 1.0 \\
\hline Preterm/low birth weight & $\begin{array}{l}18 / 422 \\
(4.3 \%)\end{array}$ & $\begin{array}{l}16 / 299 \\
(5.4 \%)\end{array}$ & $\begin{array}{c}0.79 \\
(0.39-1.6)\end{array}$ & $\begin{array}{c}0.76 \\
(0.37-1.6)\end{array}$ & $\begin{array}{l}17 / 183 \\
(9.3 \%)\end{array}$ & $\begin{array}{l}17 / 536 \\
(3.2 \%)\end{array}$ & $\begin{array}{c}3.1 \\
(1.6-6.3)\end{array}$ & $\begin{array}{c}2.5 \\
(1.2-5.2)\end{array}$ \\
\hline Postterm birth/macrosomia & $\begin{array}{c}16 / 299 \\
(5.4 \%)\end{array}$ & $\begin{array}{c}16 / 299 \\
(5.4 \%)\end{array}$ & $\begin{array}{c}1.0 \\
(0.52-1.9)\end{array}$ & $\begin{array}{c}0.95 \\
(0.47-1.9)\end{array}$ & $\begin{array}{c}10 / 183 \\
(5.5 \%)\end{array}$ & $\begin{array}{l}29 / 536 \\
(5.4 \%)\end{array}$ & $\begin{array}{c}1.0 \\
(0.48-2.1)\end{array}$ & $\begin{array}{c}1.3 \\
(0.62-2.9)\end{array}$ \\
\hline \multicolumn{9}{|l|}{ Neonatal asphyxia } \\
\hline No & $\begin{array}{l}118 / 127 \\
(92.9 \%)\end{array}$ & $\begin{array}{l}107 / 114 \\
(93.9 \%)\end{array}$ & 1.0 & & $\begin{array}{c}57 / 60 \\
(95.0 \%)\end{array}$ & $\begin{array}{l}167 / 180 \\
(92.8 \%)\end{array}$ & 1.0 & \\
\hline Yes & $\begin{array}{c}9 / 127 \\
(7.1 \%)\end{array}$ & $\begin{array}{c}7 / 114 \\
(6.1 \%)\end{array}$ & $\begin{array}{c}1.2 \\
(0.42-3.2)\end{array}$ & - & $\begin{array}{c}3 / 60 \\
(5.0 \%)\end{array}$ & $\begin{array}{l}13 / 180 \\
(7.2 \%)\end{array}$ & $\begin{array}{c}2.2 \\
(0.09-51.2)\end{array}$ & - \\
\hline \multicolumn{9}{|c|}{ Infectious diseases requiring hospitalization } \\
\hline No & $\begin{array}{l}367 / 422 \\
(87.0 \%)\end{array}$ & $\begin{array}{l}261 / 301 \\
(86.7 \%)\end{array}$ & 1.0 & 1.0 & $\begin{array}{l}150 / 182 \\
(82.4 \%)\end{array}$ & $\begin{array}{l}476 / 539 \\
(88.3 \%)\end{array}$ & 1.0 & 1.0 \\
\hline Yes & $\begin{array}{r}55 / 422 \\
(13.0 \%)\end{array}$ & $\begin{array}{r}40 / 301 \\
(13.3 \%)\end{array}$ & $\begin{array}{c}1.1 \\
(0.78-1.5)\end{array}$ & $\begin{array}{c}1.1 \\
(0.66-1.7)\end{array}$ & $\begin{array}{c}32 / 182 \\
(17.6 \%)\end{array}$ & $\begin{array}{r}63 / 539 \\
(11.7 \%)\end{array}$ & $\begin{array}{c}0.51 \\
(0.08-3.2)\end{array}$ & $\begin{array}{c}1.6 \\
(0.96-2.6)\end{array}$ \\
\hline \multicolumn{9}{|c|}{ Steroid or immunosuppressive drug usage } \\
\hline No & $\begin{array}{l}446 / 452 \\
(98.7 \%)\end{array}$ & $\begin{array}{l}311 / 312 \\
(99.7 \%)\end{array}$ & 1.0 & & $\begin{array}{l}194 / 195 \\
(99.5 \%)\end{array}$ & $\begin{array}{l}560 / 566 \\
(98.9 \%)\end{array}$ & 1.0 & \\
\hline Yes & $\begin{array}{c}6 / 452 \\
(1.3 \%)\end{array}$ & $\begin{array}{c}1 / 312 \\
(0.3 \%)\end{array}$ & $\begin{array}{c}4.2 \\
(0.50-34.9)\end{array}$ & - & $\begin{array}{c}1 / 195 \\
(0.5 \%)\end{array}$ & $\begin{array}{c}6 / 566 \\
(1.1 \%)\end{array}$ & $\begin{array}{c}4.7 \\
(0.18-122.3)\end{array}$ & - \\
\hline \multicolumn{9}{|l|}{$\geq 1$ month stay abroad } \\
\hline No & $\begin{array}{l}397 / 416 \\
(95.4 \%)\end{array}$ & $\begin{array}{l}292 / 299 \\
(97.7 \%)\end{array}$ & 1.0 & 1.0 & $\begin{array}{l}172 / 183 \\
(94.0 \%)\end{array}$ & $\begin{array}{l}515 / 531 \\
(97.0 \%)\end{array}$ & 1.0 & 1.0 \\
\hline Yes & $\begin{array}{l}19 / 416 \\
(4.6 \%)\end{array}$ & $\begin{array}{c}7 / 299 \\
(2.3 \%)\end{array}$ & $\begin{array}{c}2.0 \\
(0.83-4.8)\end{array}$ & $\begin{array}{c}1.7 \\
(0.58-4.7)\end{array}$ & $\begin{array}{l}11 / 183 \\
(6.0 \%)\end{array}$ & $\begin{array}{c}16 / 531 \\
(3.0 \%)\end{array}$ & $\begin{array}{c}0.65 \\
(0.04-11.9)\end{array}$ & $\begin{array}{c}2.5 \\
(0.95-6.6)\end{array}$ \\
\hline \multicolumn{9}{|l|}{ Siblings } \\
\hline Yes & $\begin{array}{l}381 / 424 \\
(89.9 \%)\end{array}$ & $\begin{array}{l}258 / 301 \\
(85.7 \%)\end{array}$ & 1.0 & 1.0 & $\begin{array}{l}148 / 181 \\
(81.8 \%)\end{array}$ & $\begin{array}{l}507 / 561 \\
(90.4 \%)\end{array}$ & 1.0 & 1.0 \\
\hline No & $\begin{array}{r}43 / 424 \\
(10.1 \%)\end{array}$ & $\begin{array}{r}43 / 301 \\
(14.3 \%)\end{array}$ & $\begin{array}{c}0.68 \\
(0.43-1.1)\end{array}$ & $\begin{array}{c}0.72 \\
(0.44-1.2)\end{array}$ & $\begin{array}{r}33 / 181 \\
(18.2 \%)\end{array}$ & $\begin{array}{c}54 / 561 \\
(9.6 \%)\end{array}$ & $\begin{array}{c}2.0 \\
(1.3-3.2)\end{array}$ & $\begin{array}{c}1.9 \\
(1.1-3.2)\end{array}$ \\
\hline
\end{tabular}

Values are expressed as number or odds ratio.

Sample sizes vary between variables because of missing data.

*Adjusted for sex, age, birth place, gestational week/birth weight, infectious diseases requiring hospitalization, $\geq 1$ month stay abroad, and presence of siblings.

${ }^{\top}$ Cities with $\geq 500,000$ inhabitants.

$\ddagger$ CI, confidence interval. 


\section{Acknowledgments}

The authors sincerely thank Prof. N. Tsuboyama, Prof. S. Kinoshita, Prof. M. Suzuki, Dr. Y. Maeda, Mr. N. Matsui, Dr. M. Sakuma, Ms. Y. Kubota, Mr. J. Itoh, Prof. A. Akaike, Dr. Y. Yano, Ms. N. Yonezawa, and Mr. H. Terakawa for setting up research fields and Dr. T. Kitamura, Prof. Y. Nakamura, Prof. C. Misago, Dr. T. Ihara, and Dr. Y. Takeuchi for providing us with helpful advice.

This study was not supported by any external funds.

\section{Conflict of Interest}

The authors declare no conflict of interest.

\section{References}

Allred, N.J., Shaw, K.M., Santibanez, T.A., Rickert, D.L. \& Santoli, J.M. (2005) Parental vaccine safety concerns: results from the National Immunization Survey, 2001-2002. Am. J. Prev. Med., 28, 221-224.

Bardenheier, B.H., Yusuf, H.R., Rosenthal, J., Santoli, J.M., Shefer, A.M., Rickert, D.L. \& Chu, S.Y. (2004) Factors associated with underimmunization at 3 months of age in four medically underserved areas. Public Health Rep., 119, 479-485.

Centers for Disease Control and Prevention (2009) Progress toward the 2012 measles elimination goal: Western Pacific Region, 1990-2008. MMWR Morb. Mortal. Wkly. Rep., 58, 669-673.

Gust, D.A., Darling, N., Kennedy, A. \& Schwartz, B. (2008) Parents with doubts about vaccines: which vaccines and reasons why. Pediatrics, 122, 718-725.

Holt, E., Guyer, B., Hughart, N., Keane, V., Vivier, P., Ross, A. \& Strobino, D. (1996) The contribution of missed opportunities to childhood underimmunization in Baltimore. Pediatrics, 97 , 474-480.

Manago, K., Yoshinaga, M., Nishi, J., Miyanohara, H., Maeno, N. \& Oda, H. (2004) The positive rate of antibodies against measles, chickenpox, rubella, and mumps in medical students, and a study about the availability of questionnaires as measures against hospital infection. Kankyoukansen, 19, 471474 (in Japanese).

Marks, J.S., Halpin, T.J., Irvin, J.J., Johnson, D.A. \& Keller, J.R. (1979) Risk factors associated with failure to receive vaccinations. Pediatrics, 64, 304-309.

Morrow, A.L., Rosenthal, J., Lakkis, H.D., Bowers, J.C.,
Butterfoss, F.D., Crews, R.C. \& Sirotkin, B. (1998) A population-based study of access to immunization among urban Virginia children served by public, private, and military health care systems. Pediatrics, 101, E5.

Nakamura, Y. (2010) Maternal and Child Health Handbook in Japan. JMAJ, 53, 259-265.

Osaki, K., Hattori, T., Kosen, S. \& Singgih, B. (2009) Investment in home-based maternal, newborn and child health records improves immunization coverage in Indonesia. Trans. R. Soc. Trop. Med. Hyg., 103, 846-848.

Reich, M.R., Takemi, K., Roberts, M.J. \& Hsiao, W.C. (2008) Global action on health systems: a proposal for the Toyako G8 summit. Lancet, 371, 865-869.

Rosenstock, I.M, Strecher, V.J. \& Becker, M.H. (1988) Social learning theory and the Health Belief Model. Health Educ. Q., 15, 175-183.

Smith, P.J., Humiston, S.G., Marcuse, E.K., Zhao, Z., Dorell, C.G., Howes, C. \& Hibbs, B. (2011) Parental delay or refusal of vaccine doses, childhood vaccination coverage at 24 months of age, and the Health Belief Model. Public Health Rep., 126 , 135-146.

Suarez, L., Simpson, D.M. \& Smith, D.R. (1997) Errors and correlates in parental recall of child immunizations: effects on vaccination coverage estimates. Pediatrics, 99, E3.

Takayanagi, K., Iwasaki, S. \& Yoshinaka, Y. (1993) The role of the Maternal and Child Health Handbook system in reducing perinatal mortality in Japan. Clin. Perform. Qual. Health Care, 1, 29-33.

Takeuchi, J., Goto, M., Kawamura, T. \& Hiraide, A. (2013) Influence of Vaccination dose and clinico-demographical factors on antibody titers against measles, rubella, mumps, and varicellazoster viruses among university students in Japan. Jpn. J. Infect. Dis., 66, 497-502.

Takeuchi, J., Goto, M., Kawamura, T. \& Hiraide, A. (2014) Serological assessment of measles-rubella vaccination catch-up campaign among university students. Pediatr. Int., 56, 395-399.

Wood, D., Donald-Sherbourne, C., Halfon, N., Tucker, M.B., Ortiz, V., Hamlin, J.S., Duan, N., Mazel, R.M., Grabowsky, M., Brunell, P. \& Freeman, H. (1995) Factors related to immunization status among inner-city Latino and African-American preschoolers. Pediatrics, 96, 295-301.

Yusuf, H., Adams, M., Rodewald, L., Lu, P., Rosenthal, J., Legum, S.E. \& Santoli, J. (2002) Fragmentation of immunization history among providers and parents of children in selected underserved areas. Am. J. Prev. Med., 23, 106-112. 